This information is current as of April 25, 2023.
Does the Addition of a "Black Bone" Sequence to a Fast Multisequence Trauma MR Protocol Allow MRI to Replace CT after Traumatic Brain Injury in Children?

M.H.G. Dremmen, M.W. Wagner, T. Bosemani, A. Tekes, D. Agostino, E. Day, B.P. Soares and T.A.G.M. Huisman

AJNR Am J Neuroradiol 2017, 38 (11) 2187-2192 doi: https://doi.org/10.3174/ajnr.A5405 http://www.ajnr.org/content/38/11/2187 


\title{
Does the Addition of a "Black Bone" Sequence to a Fast Multisequence Trauma MR Protocol Allow MRI to Replace CT after Traumatic Brain Injury in Children?
}

\author{
(D) M.H.G. Dremmen, DM.W. Wagner, (D)T. Bosemani, (D) A. Tekes, (DD. Agostino, (D)E. Day, DB.P. Soares, and (D).A.G.M. Huisman
}

\begin{abstract}
BACKGROUND AND PURPOSE: Head CT is the current neuroimaging tool of choice in acute evaluation of pediatric head trauma. The potential cancer risks of CT-related ionizing radiation should limit its use in children. We evaluated the role of MR imaging, including a "black bone" sequence, compared with CT in detecting skull fractures and intracranial hemorrhages in children with acute head trauma.
\end{abstract}

MATERIALS AND METHODS: We performed a retrospective evaluation of 2D head CT and brain MR imaging studies including the black bone sequence of children with head trauma. Two experienced pediatric neuroradiologists in consensus created the standard of reference. Another pediatric neuroradiologist blinded to the diagnosis evaluated brain MR images and head CT images in 2 separate sessions. The presence of skull fractures and intracranial posttraumatic hemorrhages was evaluated. We calculated the sensitivity and specificity of $\mathrm{CT}$ and MR imaging with the black bone sequence in the diagnosis of skull fractures and intracranial hemorrhages.

RESULTS: Twenty-eight children (24 boys; mean age, 4.89 years; range, 0-15.5 years) with head trauma were included. MR imaging with the black bone sequence revealed lower sensitivity (66.7\% versus $100 \%)$ and specificity ( $87.5 \%$ versus $100 \%)$ in identifying skull fractures. Four of 6 incorrectly interpreted black bone MR imaging studies showed cranial sutures being misinterpreted as skull fractures and vice versa.

CONCLUSIONS: Our preliminary results show that brain MR imaging complemented by a black bone sequence is a promising nonionizing alternative to head CT for the assessment of skull fractures in children. However, accuracy in the detection of linear fractures in young children and fractures of aerated bone remains limited.

ABBREVIATIONS: NPV = negative predictive value; $P P V=$ positive predictive value

$\mathbf{C}_{\mathrm{e}}^{\mathrm{T}}$ T is the initial neuroimaging technique of choice for the acute evaluation of pediatric head trauma due to its wider availability, lower cost, and short acquisition time. In addition, CT identifyies most traumatic injuries that require urgent treatment and correlates well with clinical scales and outcome. ${ }^{1}$ However, CTrelated ionizing radiation involves the potential risk of patients developing cancer and strongly argues in favor of alternative neuroimaging techniques such as MR imaging. ${ }^{2}$ The lifetime cancer mortality risk attributable to the radiation from a single CT scan of the head in a 1 -year-old child has been estimated as $0.07 \%$. This

Received September 6, 2016; accepted after revision June 10, 2017.

From the Section of Pediatric Neuroradiology (M.H.G.D., M.W.W., T.B., A.T., D.A. E.D., B.P.S., T.A.G.M.H.), Division of Pediatric Radiology and Pediatric Neuroradiology, Department of Radiology and Radiological Science, Johns Hopkins Hospital, Baltimore, Maryland; Division of Pediatric Radiology (M.H.G.D.), Department of Radiology, Erasmus University Medical Center Rotterdam, Rotterdam, the Netherlands; and Institute of Diagnostic and Interventional Radiology (M.W.W.), University Hospital Zurich, Zurich, Switzerland.

Please address correspondence to Marjolein H.G. Dremmen, MD, Department of Radiology, Erasmus University Medical Center Rotterdam, PO Box 2040, 3000 CA Rotterdam, the Netherlands; e-mail: m.dremmen@erasmusmc.nl

http://dx.doi.org/10.3174/ajnr.A5405 small risk translates into a large population-level risk, especially because head trauma in children from 0 to 14 years of age accounts for nearly half a million emergency department visits in the United States annually. ${ }^{3,4}$

MR imaging is a nonionizing technique that provides superior contrast resolution and has a higher sensitivity and specificity for parenchymal lesions compared with CT. ${ }^{3,4}$ Especially, advanced MR imaging techniques (DWI, SWI) provide additional information that correlates well with outcome. ${ }^{5,6}$ Nonhemorrhagic shear injuries and subtle microhemorrhages are typically seen with higher sensitivity by MR imaging compared with CT. Nevertheless, the role of MR imaging in the acute diagnostic work-up of head trauma in children is still limited. ${ }^{2,7,8}$ This limitation may be partially explained by longer acquisition times and the subsequent need for sedation as well as the low sensitivity of MR imaging for skull fractures. ${ }^{2,8}$ Recently, black bone MR images have been introduced as a new sequence for the evaluation of structural bony abnormalities such as craniosynostosis. ${ }^{9}$

On the basis of the inherent diagnostic quality of the black bone sequence, we aimed to determine whether a trauma brain 
MR imaging protocol with an included black bone MR image could be an alternative to head CT in the acute work-up of children with head trauma. To address our goal, we compared the diagnostic accuracy of brain MR imaging including the black bone sequence with CT for the detection of skull fractures after traumatic brain injury in children. Images were also studied for coexisting intracranial lesions.

\section{MATERIALS AND METHODS}

This single-center retrospective study was approved by the Johns Hopkins Hospital institutional research ethics board, which waived informed consent.

\section{Study Population}

Inclusion criteria were the following: 1) a history of head trauma; 2) the availability of thin-section ( $\leq 3 \mathrm{~mm}$ ) 2D head CT data and a brain MR imaging study including a black bone sequence acquired within 7 days of each other, and 3) age at neuroimaging younger than 18 years. The exclusion criteria were the following: 1) susceptibility artifacts on a black bone sequence due to implanted materials, and 2) a brain MR imaging study acquired after craniotomy. Data from eligible children were obtained through an electronic search of our pediatric neuroradiology data base covering the period between January 1, 2015 (date when black bone MR imaging was introduced in our hospital), and November 15, 2015.

\section{Image Acquisition}

All CT studies performed at our radiology department were acquired on a commercially available $2 \times 128$ detector system (Somatom Definition Flash; Siemens, Erlangen, Germany) using the institutional pediatric head CT protocol, including the following parameters: tube voltage, $120 \mathrm{kV}$; tube current, 380 reference $\mathrm{mA}$; rotation time, 1.0 second; axial acquisition; $0.75-\mathrm{mm}$ section thickness; FOV , $160 \times 160$ to $250 \times 250 \mathrm{~mm}$. No intravenous injection of a contrast agent was performed. The CT studies imported to our neuroradiology data base from outside hospitals and officially reviewed by our pediatric neuroradiologists met the inclusion criteria if the studies were performed with a section thickness of $\leq 3 \mathrm{~mm}$.

The brain MR imaging studies were performed on a $1.5 \mathrm{~T}$ (Magnetom Aera; Siemens) or 3T (Magnetom Skyra; Siemens) clinical scanner using a standard pediatric 16-channel head coil. The institutional pediatric head trauma MR imaging protocol includes a 3D T1-weighted sequence with a sagittal acquisition, an axial T2-weighted sequence, axial FLAIR, DTI, and SWI. In addition, a black bone sequence was acquired in the axial plane from above the skull vertex to below the mandible, with $2 \mathrm{D}$ reconstruction in the coronal and sagittal planes. The black bone sequence is a gradient-echo sequence with short TEs and TRs and an optimal flip angle for the differentiation of bone from soft-tissue contrast (TE, $4.20 \mathrm{~ms}$; TR, $8.60 \mathrm{~ms}$; flip angle, $5^{\circ}$; FOV, $240 \mathrm{~mm}$; section thickness, $1.00 \mathrm{~mm}$; section spacing, $0.2 \mathrm{~mm}) .{ }^{10}$ Signal from fat and water is suppressed to provide uniform soft-tissue contrast, thereby optimizing the visualization of the bone-soft tissue margin. ${ }^{10,11}$

\section{Image Analysis}

Both the CT and MR imaging datasets were evaluated for the presence of skull fractures and intracranial hemorrhages. Addi- tionally, the fracture type was classified into linear, depressed, or basilar, and the type of intracranial hemorrhage was subdivided into subdural, epidural, subarachnoid, intraparenchymal, intraventricular, and mixed ( $>1$ type of intracranial hemorrhage). ${ }^{3,4}$

The standard of reference for the diagnosis of a skull fracture and/or posttraumatic intracranial hemorrhage was established by consensus interpretation of 2 experienced pediatric neuroradiologists with 14 and 22 years of experience (A.T. and T.A.G.M.H.). The complete CT datasets, including $2 \mathrm{D}$ and, if available, $3 \mathrm{D}$ images, and the full MR imaging datasets were reviewed to create the standard of reference.

The study reader was a pediatric neuroradiologist with 7 years of experience (T.B.). The reader was blinded to the medical records, clinical examination findings, previous neuroimaging studies, and final diagnosis. In a first reading session, he evaluated the available MR imaging studies of all patients in a random order. The reader first assessed the presence, location, and type of skull fractures using only the black bone sequence. After this initial assessment, the reader could use the other available sequences to identify posttraumatic intracranial lesions. No adjustments were made to the black bone interpretation score after the assessment of the intracranial lesions. In a second independent reading session 30 days after the first session, the 2D-CT studies were evaluated in a different random order. The first author (M.H.G.D.) assisted the study reader by opening only the relevant images for each reading session and entering the results of the evaluation on an anonymized data sheet. The black bone images were reviewed in regular and inverted format. Empirically, the inverted MR imaging window level setting resembles the classic bone CT appearance best.

\section{Statistical Analysis}

The independent evaluations of the CT images and MR imaging datasets (including the black bone sequence) from the study reader were compared with the standard of reference to analyze the diagnostic accuracy. To compare the CT and MR imaging data for the presence of skull fractures and intracranial hemorrhages, we used $2 \times 2$ contingency tables. The decision to report the presence or absence of skull fractures or intracranial hemorrhages was considered as, respectively, a true-positive or true-negative result when it matched the consensus reading of the standard of reference or as, respectively, a false-positive or a false-negative result when it did not match the reading of the standard of reference. To calculate sensitivity, specificity, positive predictive value (PPV), and negative predictive value (NPV), we correlated the specific decisions (true-positive, false-positive, true-negative, or false-negative) with the total decisions for the study reader. For both 2D head CT and brain MR imaging including the black bone sequence, the sensitivity, specificity, PPV, and NPV were calculated for the detection of skull fractures only, intracranial hemorrhages only, and skull fractures and/or intracranial hemorrhages combined (representing whether the neuroimaging study findings were normal for fractures and hemorrhages). Finally, the sensitivity, specificity, PPV, and NPV of brain MR imaging including the black bone sequence in the detection of skull fractures were calculated for 2 different age groups, children up to 2 years of age (with relatively wide cranial sutures) and older than 2 years of 
age, and for children with MR imaging studies performed on 1.5T and 3T MR imaging scanners, respectively.

\section{RESULTS}

\section{Study Population}

Twenty-eight children (24 boys and 4 girls) with acute head trauma met the inclusion criteria for this study. The mean age of the children at the first neuroimaging study was 4.89 years (range, 6 days to 15.5 years). None of the patients died. Fourteen children (50\%) were younger than of 2 years of age. The field strength of the MR imaging scanner was $1.5 \mathrm{~T}$ in 12 children and $3 \mathrm{~T}$ in 16 children. For 7 children (25\%), anesthesia was necessary to perform MR imaging. The mean time interval between the CT and MR imaging was $2 \pm 1.43$ days (range, $0-6$ days).

\section{Imaging Analysis}

On the basis of the standard of reference, 12 children had skull fractures (43\%) and 22 children (79\%) had intracranial hemorrhages. The different types of skull fractures and intracranial hemorrhages are shown in Table 1 . Six children with a skull fracture were younger than 2 years of age. Twenty-two of 28 children $(79 \%)$ had at least a skull fracture and/or an intracranial hemorrhage (all children with a skull fracture had an intracranial hemorrhage).

The sensitivity, specificity, PPV, and NPV for 2D head CT and brain MR imaging including the black bone sequence for detecting skull fractures, intracranial hemorrhages, and skull fractures and/or intracranial hemorrhages combined are shown in Table 2. The 2D head CT had a higher sensitivity, specificity, PPV, and NPV compared with brain MR imaging including the black bone sequence for the detection of skull fractures, while brain MR imaging including the black bone sequence had a higher sensitivity,

\begin{tabular}{|c|c|c|}
\hline & $\begin{array}{l}\text { No. of } \\
\text { Patients }\end{array}$ & $\begin{array}{l}\text { Percentage } \\
\text { of Patients }\end{array}$ \\
\hline Skull fractures & 12 & $43 \%$ \\
\hline Linear & 11 & $39 \%$ \\
\hline Depressed & 1 & $4 \%$ \\
\hline Intracranial hemorrhage & 22 & $79 \%$ \\
\hline Isolated subdural & 4 & $14 \%$ \\
\hline Isolated epidural & 3 & $11 \%$ \\
\hline Isolated intraparenchymal & 3 & $11 \%$ \\
\hline Mixed & 12 & $43 \%$ \\
\hline
\end{tabular}

\begin{tabular}{|c|c|c|c|c|}
\hline & \multirow{2}{*}{$\begin{array}{c}\text { Skull Fractures } \\
\text { MRI + BB } \\
\text { (Compared with CT) }\end{array}$} & \multirow{2}{*}{$\begin{array}{c}\begin{array}{c}\text { Intracranial } \\
\text { Hemorrhages }\end{array} \\
\text { Axial CT } \\
\text { (Compared with MRI) }\end{array}$} & \multicolumn{2}{|c|}{$\begin{array}{c}\text { Skull Fracture } \\
\text { and/or Intracranial } \\
\text { Hemorrhages }\end{array}$} \\
\hline & & & Axial CT & MRI + BB \\
\hline Sensitivity & $66.7 \%$ & $72.7 \%$ & $81.8 \%$ & $100 \%$ \\
\hline Specificity & $87.5 \%$ & $83.3 \%$ & $83.3 \%$ & $100 \%$ \\
\hline PPV & $80.0 \%$ & $94.1 \%$ & $94.7 \%$ & $100 \%$ \\
\hline NPV & $77.8 \%$ & $45.5 \%$ & $55.6 \%$ & $100 \%$ \\
\hline
\end{tabular}

Note:-BB indicates black bone MRI sequence. specificity, PPV, and NPV compared with 2D head CT for the detection of skull fractures and/or intracranial hemorrhages combined.

The sensitivity, specificity, PPV, and NPV for brain MR imaging including the black bone sequence in detecting skull fractures dependent on the age of the children at neuroimaging and the magnetic field strength of the MR scanner are shown in Table 3. In children younger than 2 years of age, the sensitivity and NPV for brain MR imaging including the black bone sequence in detecting skull fractures were higher and the specificity and PPV were lower compared with children older than 2 years of age. In children with brain MR imaging acquired on a 1.5T MR imaging scanner, the sensitivity, PPV, and NPV for brain MR imaging including the black bone sequence in detecting skull fractures were higher and the specificity was lower compared with children with brain MR imaging acquired on a 3T MR imaging scanner.

\section{DISCUSSION}

Skull fractures are relatively common in children with head trauma (up to $30 \%$ ), particularly in the younger age groups. ${ }^{12,13}$ Morphologically, skull fractures can be described as linear, depressed, or basilar (skull base). Most skull fractures are linear $(66 \%-75 \%){ }^{14,15}$ Although isolated fractures themselves rarely require intervention, a neuroimaging study may be needed to describe the full extent of calvarial and intracranial injuries. ${ }^{16}$

Black bone is a novel MR imaging sequence that uses short TEs and TRs as well as an optimal flip angle to minimize soft-tissue contrast and enhance the bone-soft-tissue boundary. ${ }^{9-11,17}$ Signal from fat and water is suppressed to provide uniform soft-tissue contrast, thereby optimizing the visualization of the bone-soft-tissue interface. ${ }^{10,11}$ The short TEs and TRs and the volume acquisition result in short imaging times. Black bone MR imaging has been shown to be useful and accurate in the evaluation of cranial bones and sutures in children with craniosynostosis. This finding reveals a considerable clinical potential in the assessment of osseous abnormalities as a nonionizing alternative to CT. ${ }^{9-11,17}$

Our study evaluated the diagnostic accuracy of black bone MR imaging in detecting skull fractures in children with head trauma compared with CT. The black bone MR imaging sequence showed a sensitivity of $66.7 \%$ and a specificity of $87.5 \%$ in the detection of skull fractures (Fig 1). The specificity is acceptable, while the sensitivity is low. To determine the reasons for the low sensitivity of the black bone MR imaging sequence, we studied the 4 falsenegative linear fractures and the 2 false-positive linear fractures. In 2 children younger than 2 years of age, false-negative linear fractures were misinterpreted as cranial sutures ( 2 false-negative cases). Furthermore, the 2 false-positive cases were children in the younger age group (younger than 2 years), with cranial sutures being falsely identified as linear fractures. In young children, the presence of open sutures is known to increase the diagnostic uncertainty of skull fractures on 2D images as recently shown by a head CT study. ${ }^{14}$ In head CT studies, the addition of $3 \mathrm{D}$ reconstructions to the $2 \mathrm{D}$ dataset has been shown 
to increase the reader's confidence for correct differentiation of sutures and other nonfracture-related linear lucencies such as vascular channels versus linear fractures. ${ }^{14}$ The addition of $3 \mathrm{D}$ reconstruction to the 2D black bone MR images as shown for the study of craniosynostosis ${ }^{9}$ could potentially decrease the misinterpretation as shown for head CT.

The third false-negative case was an older child in whom a linear fracture was misinterpreted as a venous transosseous vascular channel. More familiarity with the black bone sequence and the use of $3 \mathrm{D}$ reconstruction could potentially help overcome this problem. The fourth case of a missed linear fracture on black bone MR imaging was a fracture in the mastoid region (Fig 2). This case highlights the main limitation of black bone MR imaging: In anatomic regions where bone abuts air (mastoid, craniofacial sinuses), the distinction between air and bone is difficult because both have low signal intensity on this sequence. ${ }^{10}$ The application of black bone MR imaging at air-bone interfaces is challenging

Table 3: Differences in diagnostic accuracy of brain MRI including the black bone sequence for the detection of skull fractures in 28 children with head trauma, depending on the age of the child and MR imaging field strength

\begin{tabular}{|c|c|c|c|c|}
\hline & \multicolumn{2}{|c|}{ Age at MRI } & \multicolumn{2}{|c|}{$\begin{array}{l}\text { MRI Field } \\
\text { Strength }\end{array}$} \\
\hline & $\begin{array}{c}\text { Younger } \\
\text { Than } 2 \text { yr } \\
(n=14)\end{array}$ & $\begin{array}{c}2 \text { yr and } \\
\text { Older } \\
(n=14)\end{array}$ & $\begin{array}{c}1.5 T \\
(n=12)\end{array}$ & $\begin{array}{c}3 \mathrm{~T} \\
(n=16)\end{array}$ \\
\hline Sensitivity & $75.0 \%$ & $50.0 \%$ & $83.3 \%$ & $50.0 \%$ \\
\hline Specificity & $66.7 \%$ & $100 \%$ & $83.3 \%$ & $90.0 \%$ \\
\hline PPV & $75.0 \%$ & $100 \%$ & $83.3 \%$ & $75.0 \%$ \\
\hline NPV & $66.7 \%$ & $83.3 \%$ & $83.3 \%$ & $75.0 \%$ \\
\hline
\end{tabular}

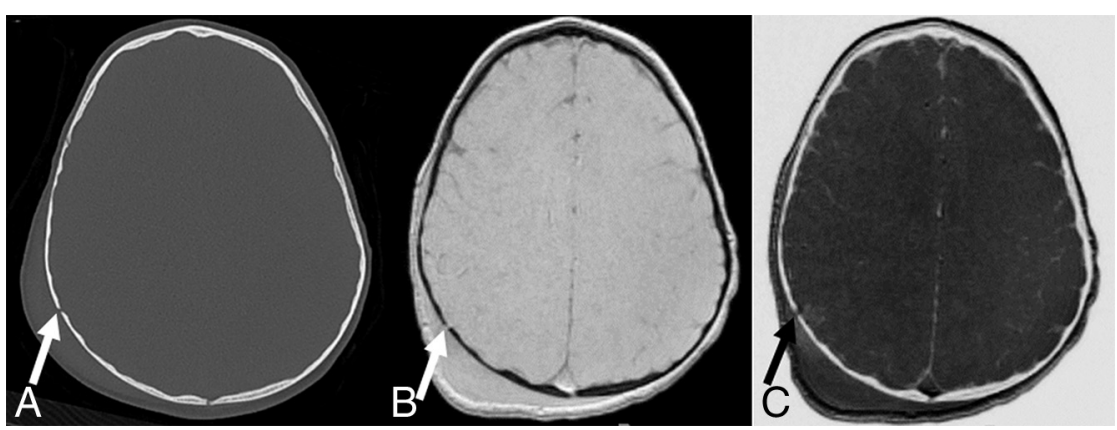

FIG 1. A, Axial CT image shows a nondisplaced linear fracture of the right parietal bone (arrow) with extracranial soft-tissue swelling. Black bone $(B)$ and inverted black bone $(C)$ MR images reveal equivalent visualization of the right parietal fracture (arrows), as well as overlying soft-tissue swelling.

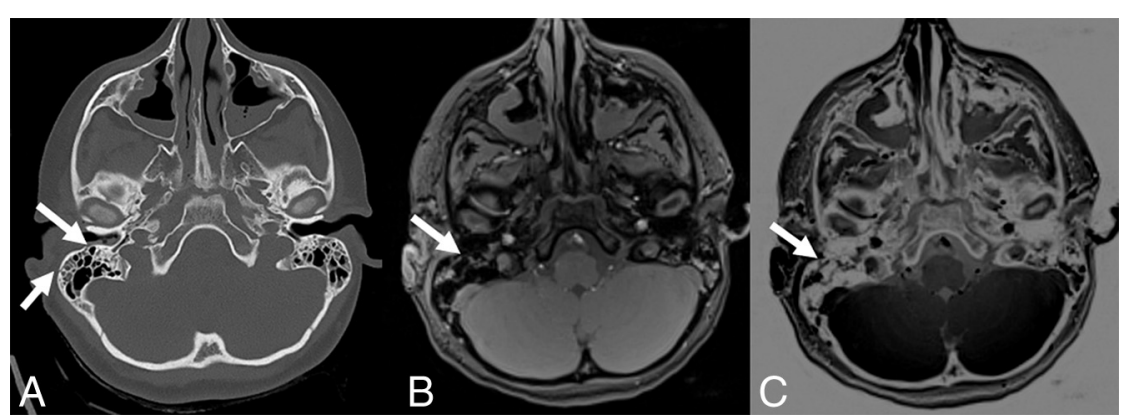

FIG 2. A, Axial CT image shows 2 small nondisplaced linear fractures of the mastoid (arrows). On black bone $(B)$ and inverted black bone $(C)$ MR images, these fractures are barely visible (arrows). and requires experience with the technique and careful review of the images.

The fractures missed on black bone MR images were linear and nondisplaced and did not require specific treatment. Fractures of the mastoid air cells and temporal bone region are potentially of major clinical concern; in case of suspected CSF leaks, damage to inner/middle ear structures, and vascular injury, CT is needed. Further improvement of the black bone imaging technique is necessary for implementation of this technique to all neurotrauma patients. Isolated, linear, nondisplaced skull fractures are known to have a low clinical significance: They are not associated with neurologic deterioration, affected children can be appropriately managed as outpatients, and neurologic outcome is excellent. ${ }^{2,18}$ A precise and complete diagnosis in children with head trauma is important (eg, for medical legal purposes); therefore for the work-up of nonaccidental trauma or for other carefully selected pediatric patients, an additional head CT study may be necessary if the black bone MR images are negative for identification of skull fractures. In most cases, the missed diagnosis of an isolated, linear skull fracture appears to have low clinical significance and no impact on the management of the affected children.

By using MR imaging including the black bone sequence as a primary screening tool in patients with neurotrauma, the number of CT studies in the pediatric population can be decreased. The identification of intracranial hemorrhages, on the other hand, has important implications for acute management and long-term outcome of children with head trauma. ${ }^{3,4,19}$ Our study reveals a higher sensitivity (100\% versus $72.7 \%$ ) and specificity (100\% versus $83.3 \%$ ) of MR imaging including black bone sequence compared with head CT in detecting intracranial hemorrhages in children with head trauma (Fig 3). The higher sensitivity and specificity of MR imaging is not surprising: The superior contrast resolution of MR imaging compared with CT results in a higher sensitivity for parenchymal lesions. ${ }^{20}$ The implementation of SWI as part of the MR imaging protocol for children with head trauma further increases the sensitivity for detection of intracranial hemorrhages. Additionally, a strong correlation has been shown between the number and volume of SWI lesions and the severity of injury (determined by the initial Glasgow Coma Scale score and the duration of the coma) as well as neurologic outcome 6-12 months after the injury. ${ }^{21}$ These results emphasize the importance of MR imaging in the acute work-up of children with head trauma. In our opinion, MR imaging as a nonionizing imaging technique should not only be an important alternative to CT in the acute evaluation of pediatric head trauma but also gradually become the main neuroimaging technique for the evaluation of trau- 


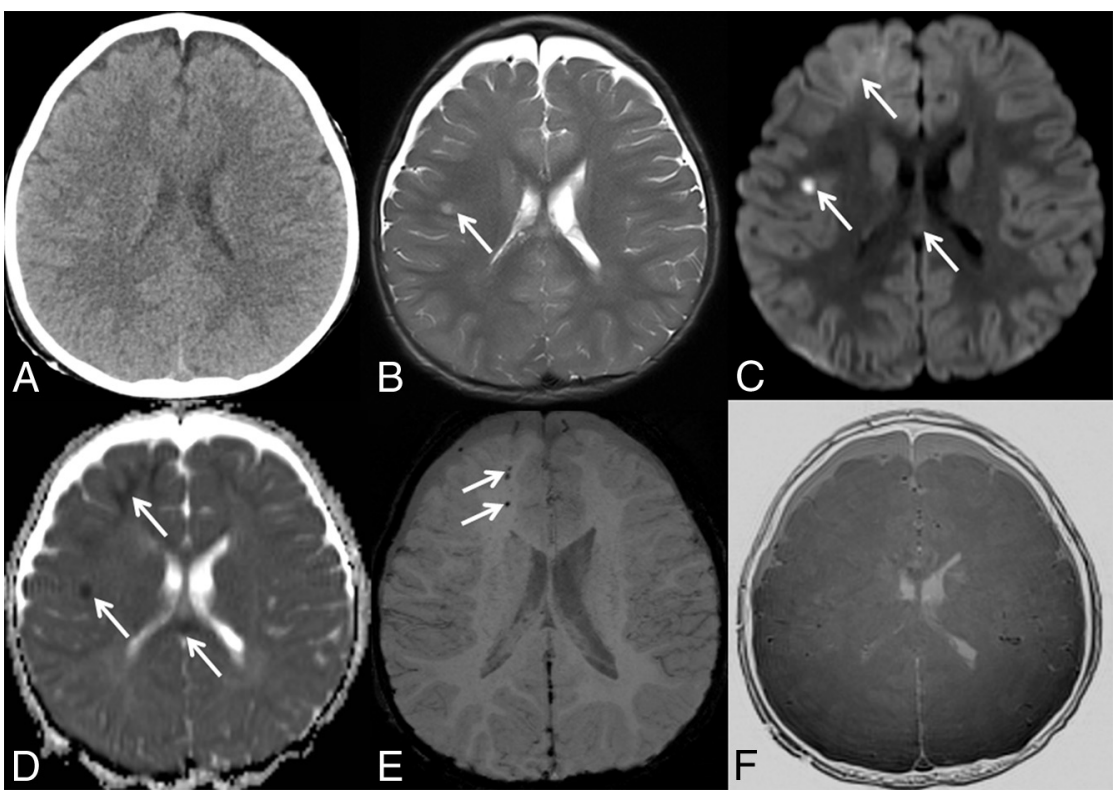

FIG 3. $A$, Axial $C T$ image does not show intracranial hemorrhage. A matching axial T2-weighted MR image $(B)$, axial trace of diffusion $(C)$, ADC map $(D)$, minimal intensity projection-SWI $(E)$, and inverted black bone MR image $(F)$ reveal areas of T2-hyperintense signal and restricted diffusion within the temporal white matter (arrows in $B-D$ ), areas of restricted diffusion within the right frontal lobe and splenium of the corpus callosum (arrows in $C$ and $D$ ), and foci of hypointense SWI signal within the right frontal white matter (arrows in E), suggestive of intracranial hemorrhages and diffuse axonal injury not seen on axial CT.

matic skull and brain injury in the pediatric population. Specifically, the combination of highly sensitive MR imaging sequences for identification of intracranial and intra-axial lesions with a relatively sensitive MR image for clinically relevant fractures offers a diagnostically important "one-stop shopping” imaging approach. In our institution, we currently use a fast trauma head MR imaging, which combines the black bone MR image with 3D T1-weighted, axial HASTE T2-weighted, axial DWI, and SWI sequences.

Our results show a negative correlation between the sensitivity of black bone MR imaging and the magnetic field strength of the MR imaging scanner: $83 \%$ in $1.5 \mathrm{~T}$ versus $50 \%$ in $3 \mathrm{~T}$. The short TE and TR cause the black bone sequence to be a gradient-echo sequence, hence susceptible to artifacts. Movement artifacts, dental braces, and implanted materials (ie, ventriculoperitoneal shunt reservoirs) can potentially degrade image quality and decrease the sensitivity of the study, particularly at 3T due to higher amenability to susceptibility artifacts in $3 \mathrm{~T}$ datasets. In addition, our study reveals a lower specificity for black bone MR imaging in children younger than 2 years of age compared with older children. This difference is most likely due to the confounding role of open cranial sutures in younger children as shown for head CT data. ${ }^{14}$

We acknowledge the limitations of our study, including the small number of patients and its retrospective nature. In addition, the standard of reference was established by experienced pediatric neuroradiologists using all available images (CT and MR imaging), as is typical in daily routine. We had no postmortem studies because all patients survived. Furthermore, head CT and brain MR imaging data were not acquired on the same day in some patients. The optimal study design to compare the diagnostic accuracy of CT and MR imaging datasets would require the acqui- sition of CT and MR imaging studies on the same day. However, in this retrospective study, this was not feasible due to both practical and ethical reasons. Future prospective research should focus on optimizing the study design to avoid selection bias. For 7 children, anesthesia was necessary to perform brain MR imaging. This was partly because most of the study population was younger than 6 months of age or older than 5 years and the MR imaging examination could be performed with the patient in a vacuum cushion or after preparing and coaching the child and the parents/caregivers, respectively. The increasing availability of child life specialists may further help decrease the need for anesthesia for brain MR imaging in the future. Finally, brain MR imaging examinations were obtained for clinical indications on the basis of the head CT findings or neurologic symptoms not explained by the head CT findings. This choice may have been a potential source of selection bias in our study population. A prospective study design in which CT and MR imaging studies are performed in all pediatric trauma patients is needed for further evaluation of MR imaging as a primary neuroimaging screening tool in the pediatric population.

\section{CONCLUSIONS}

Our preliminary results show that brain MR imaging including the black bone sequence may be a promising alternative to head $\mathrm{CT}$ as a primary screening tool for the acute diagnostic work-up of children with head trauma. The higher sensitivity and specificity of MR imaging in detecting intracranial hemorrhages compared with CT highlights the key role of MR imaging for acute management and prognosis of long-term outcome of children with head trauma. The lack of ionizing radiation further supports the use of brain MR imaging as the primary neuroimaging tool for acute head trauma in the pediatric population. Currently, additional head CT studies are indicated for patients without identifiable skull fracture on the black bone sequence because of the possibility of missing certain types of skull fractures. Prospective studies with a larger number of children are needed to further evaluate the diagnostic role of black bone MR imaging in children with acute head trauma. Furthermore, the value of a fast trauma MR imaging protocol should be prospectively evaluated for the wide range of posttraumatic lesions that may be encountered in the brain, including diffuse axonal injury, nonhemorrhagic contusions, and tissue lacerations.

\section{ACKNOWLEDGMENTS}

We thank and dedicate this study to our friend and exceptionally gifted and talented colleague Dr Andrea Poretti for providing his insight and expertise. We are immensely grateful for his dedica- 
tion to the field of pediatric neuroimaging. He left us too early, and we will miss his sharp intellect, humble character, and relentless effort to advance our understanding of pediatric neurologic diseases.

Disclosures: Marjolein H.G. Dremmen—UNRELATED: Employment: Pediatric Radiologist, Erasmus Medical Center Rotterdam, Comments: 3-month observing fellowship in pediatric neuroradiology, Johns Hopkins Hospital (unpaid). Thangamadhan Bosemani-UNRELATED: Consultancy: Alexion Pharmaceutical. Thierry A.G.M. Huisman-UNRELATED: Board Membership: Editorial Board American Journal of Neuroradiology.

\section{REFERENCES}

1. Klassen TP, Reed MH, Stiell IG, et al. Variation in utilization of computed tomography scanning for the investigation of minor head trauma in children: a Canadian experience. Acad Emerg Med 2000;7:739-44 CrossRef Medline

2. Roguski M, Morel B, Sweeney M, et al. Magnetic resonance imaging as an alternative to computed tomography in select patients with traumatic brain injury: a retrospective comparison. J Neurosurg Pediatr 2015;15:529-34 CrossRef Medline

3. Pinto PS, Poretti A, Meoded A, et al. The unique features of traumatic brain injury in children: review of the characteristics of the pediatric skull and brain, mechanisms of trauma, patterns of injury, complications and their imaging findings-part 1. J Neuroimaging 2012;22:e1-17 CrossRef Medline

4. Pinto PS, Meoded A, Poretti A, et al. The unique features of traumatic brain injury in children: review of the characteristics of the pediatric skull and brain, mechanisms of trauma, patterns of injury, complications, and their imaging findings-part 2. J Neuroimaging 2012;22:e18-41 CrossRef Medline

5. Beauchamp MH, Ditchfield M, Babl FE, et al. Detecting traumatic brain lesions in children: CT versus MRI versus susceptibility weighted imaging (SWI). J Neurotrauma 2011;28:915-27 CrossRef Medline

6. Bosemani T, Poretti A, Huisman TA. Susceptibility-weighted imaging in pediatric neuroimaging. J Magn Reson Imaging 2014;40: 530-44 CrossRef Medline

7. Schmutz B, Rahmel B, McNamara Z, et al. Magnetic resonance imaging: an accurate, radiation-free, alternative to computed tomography for the primary imaging and three-dimensional reconstruction of the bony orbit. J Oral Maxillofac Surg 2014;72:611-18 CrossRef Medline

8. Sigmund GA, Tong KA, Nickerson JP, et al. Multimodality compar- ison of neuroimaging in pediatric traumatic brain injury. Pediatr Neurol 2007;36:217-26 CrossRef Medline

9. Eley KA, Watt-Smith SR, Sheerin F, et al. "Black bone" MRI: a potential alternative to $\mathrm{CT}$ with three-dimensional reconstruction of the craniofacial skeleton in the diagnosis of craniosynostosis. Eur Radiol 2014;24:2417-26 CrossRef Medline

10. Eley KA, McIntyre AG, Watt-Smith SR, et al. "Black bone" MRI: a partial flip angle technique for radiation reduction in craniofacial imaging. Br J Radiol 2012;85:272-78 CrossRef Medline

11. Eley KA, Watt-Smith SR, Golding SJ. "Black bone" MRI: a potential alternative to $\mathrm{CT}$ when imaging the head and neck: report of eight clinical cases and review of the Oxford experience. Br J Radiol 2012; 85:1457-64 CrossRef Medline

12. Greenes DS, Schutzman SA. Clinical significance of scalp abnormalities in asymptomatic head-injured infants. Pediatr Emerg Care 2001;17:88-92 CrossRef Medline

13. Dunning J, Daly JP, Lomas JP, et al; Children's head injury algorithm for the prediction of important clinical events study group. Derivation of the children's head injury algorithm for the prediction of important clinical events decision rule for head injury in children. Arch Dis Child 2006;91:885-91 CrossRef Medline

14. Orman G, Wagner MW, Seeburg D, et al. Pediatric skull fracture diagnosis: should 3D CT reconstructions be added as routine imaging? J Neurosurg Pediatr 2015;16:426-31 CrossRef Medline

15. Schutzman SA, Greenes DS. Pediatric minor head trauma. Ann Emerg Med 2001;37:65-74 Medline

16. Bonfield CM, Naran S, Adetayo OA, et al. Pediatric skull fractures: the need for surgical intervention, characteristics, complications, and outcomes. J Neurosurg Pediatr 2014;14:205-11 CrossRef Medline

17. Eley KA, Watt-Smith SR, Golding SJ. "Black bone" MRI: a potential non-ionizing method for three-dimensional cephalometric analysis-a preliminary feasibility study. Dentomaxillofac Radiol 2013;42:20130236 CrossRef Medline

18. Powell EC, Atabaki SM, Wootton-Gorges S, et al. Isolated linear skull fractures in children with blunt head trauma. Pediatrics 2015;135: e851-57 CrossRef Medline

19. Hochstadter E, Stewart TC, Alharfi IM, et al. Subarachnoid hemorrhage prevalence and its association with short-term outcome in pediatric severe traumatic brain injury. Neurocrit Care 2014;21: 505-13 CrossRef Medline

20. Tang PH, Lim CC. Imaging of accidental paediatric head trauma. Pediatr Radiol 2009;39:438-46 CrossRef Medline

21. Tong KA, Ashwal S, Holshouser BA, et al. Diffuse axonal injury in children: clinical correlation with hemorrhagic lesions. Ann Neurol 2004;56:36-50 CrossRef Medline 